\title{
Scaling theory of quantum resistance distributions in disordered systems
}

\author{
A M JAYANNAVAR* \\ Department of Physics, Indian Institute of Science, Bangalore 560012, India \\ * Permanent address: Institute of Physics, Sachivalaya Marg. Bhubaneswar 751005 , India \\ MS received 25 January 1991; revised 3 April 1991
}

\begin{abstract}
We have derived explicitly, the large scale distribution of quantum Ohmic resistance of a disordered one-dimensional conductor. We show that in the thermodynamic limit this distribution is characterized by two independent parameters for strong disorder, leading to a two-parameter scaling theory of localization. Only in the limit of weak disorder we recover single parameter scaling, consistent with existing theoretical treatments.
\end{abstract}

Keywords. Localization; quantum Ohmic resistance; scaling; Fokker-Planck equations; invariant imbedding.

PACS Nos $72 \cdot 15 ; 72 \cdot 10 ; 72 \cdot 90 ; 71 \cdot 55$

\section{Introduction}

There have been many studies on zero temperature quantum Ohmic resistance and fluctuation of a disordered one-dimensional conductor in the absence of electron interactions [Anderson et al 1980; Abrahams and Stephen 1980; Abrikosov 1981; Melnikov 1980; Kumar 1985; Heinrichs 1986; Rammal and Doucot 1987; Shapiro 1987]. It is now well established that coherent interference effects, due to elastic scattering by the serial static disorder, lead to strong localization of electronic eigenstates for arbitrarily weak disorder [Erdos and Herndon 1982]. The localized nature of the eigenstates manifests as an exponential increase of ensemble averaged resistance $\langle\rho\rangle$ with the sample length $L$. Experiments on quasi-one-dimensional wires confirm this length-scale dependence of resistance, with the Thouless length $L_{T}=(D \tau)_{\text {in }}^{1 / 2}$ effectively replacing the sample length [Giordano 1980; Bishop and Dolan 1985; Farrell et al 1985]. In addition to being non-additive, the quantum Ohmic resistance is also known to be non-self-averaging in that the resistance fluctuations over the ensemble of macroscopically identical samples dominate the ensemble average, i.e., there is no typical resistance. In fact the relative fluctuation of resistance is much larger than the relative fluctuation of the underlying impurity concentration for large sample lengths. Resistance fluctuations get somewhat suppressed by the multiple connectivity of higher dimensions $(d>1)$. It has been shown that [Lee and Stone 1985; Altshuler and Khmelnitskii 1985; Altshuler et al 1986; Heinrichs et al 1988] in higher dimensions a metallic domain is characterized by the universal fluctuations $\left(e^{2} / h\right)$, i.e., conductance of a metal self-averages in three dimensions but the relative variance decreases more slowly than the inverse volume dependence expected classically. Several other results suggest that the fluctuations persist right up to the mobility edge on the insulating side in higher dimensions $(d>2)$ and are suspected to have an important bearing on 
the physics of the mobility edge [Kumar and Jayannavar 1986; Ioffe et al 1985; Efetov 1984; Cohen et al 1988].

The purpose of the present work is to discuss the scaling properties of the resistance distribution in one-dimensional random systems. In particular, we show that in the thermodynamic limit one obtains a limiting universal distribution. This distribution which determines the macroscopic behaviour (i.e., insensitivity to microscopic details) is characterized by two independent parameters (two-parameter scaling) in the strong disorder limit [Cohen et al 1988]. Only in the limit of weak disorder one finds a single parameter scaling [Abrahams et al 1979] in agreement with previous results. Our results are consistent with the recent study of Cohen et al [Cohen et al 1988] and we also point out the approximations made in the earlier studies that point towards a single parameter scaling [Anderson et al 1980; Abrahams and Stephen 1980; Abrikosov 1981; Melnikov 1980; Kumar 1985; Heinrichs 1986; Rammal and Doucot 1987; Shapiro 1987]. Our treatment is based on the invariant imbedding approach, applicable in any linear or nonlinear problem with serial randomness [Kumar 1985; Heinrichs 1986; Rammal and Doucot 1987; Bellman and Wing 1976; Chandrasekhar 1960].

\section{Method and calculations}

We start with the well-known Landauer formula [Landauer 1970], which expresses the residual resistance of a one-dimensional system in terms of its scattering properties at the Fermi energy. The expression for the resistance $\rho$, measured in the units of $\left(\pi \hbar / e^{2}\right)$ and including the spin is given by

$$
\rho=\frac{R(L) R^{*}(L)}{1-R(L) R(L)^{*}},
$$

where $R(L)$ is the complex amplitude reflection coefficient for the one-dimensional conductor of length $L$. The model Hamiltonian for the one-dimensional disordered system is,

$$
H=-\frac{\hbar^{2}}{2 m} \frac{\partial^{2}}{\partial x^{2}}+V(x)
$$

where $V(x)$ for $0<x<L$ is the random potential assumed to be a delta correlated Gaussian variable with,

$$
\begin{aligned}
& \langle V(x)\rangle=0, \\
& \left\langle V(x) V\left(x^{\prime}\right)\right\rangle=2 V_{0}^{2} \delta\left(x-x^{\prime}\right) .
\end{aligned}
$$

Here $V_{0}$ measures the strength of the disorder. The disordered sample extends from $x=0$ to $x=L$, the two ends being connected to perfect leads. Consider the scattering of an electron plane wave $\exp [-i k(x-L)]$ of energy $E$ incident at $x=L$ from the right $(x>L)$. It is partially reflected with the complex amplitude reflection coefficient $R(L)$ and is partially transmitted with the complex transmission coefficient $T(L)$. The general principle of the method of invariant imbedding is to address the emergent quantity, namely, $R(L)$ (or $T(L)$ ) in a form which does not involve the wave function 
inside the conductor explicitly. This is done by reducing the boundary value problem to an initial value problem relative to the imbedding parameters. The complex coefficient $R(L)$ is given by the Riccati equation [Heinrichs 1986; Rammal and Doucot 1987; Bellman and Wing 1976]

$$
i k_{0} \frac{\mathrm{d} R(L)}{\mathrm{d} L}=V(L)\left(1+R^{2}\right)-2\left(k_{0}^{2}-V(L)\right) R
$$

where $k_{0}$ is the incident wave vector of an electron and $k^{2}=E$ (we have set units such that $\left.\left(2 m / h^{2}\right)=1\right)$. It is convenient to write $R=r \exp i \theta$, where $\theta$ is the phase of the reflected wave and $r^{2}$ is the reflection coefficient. Now substituting this form for $R$ in (4) and making use of the relation for the resistance (eq. (1)), we immediately get the evolution for $\rho$ and $\theta$ as,

$$
\begin{aligned}
& \frac{\mathrm{d} \rho}{\mathrm{d} L}=-\frac{2 V(L)}{k_{0}} \sin \theta[\rho(\rho+1)]^{1 / 2}, \\
& \frac{\mathrm{d} \theta}{\mathrm{d} L}=2 k_{0}-\frac{V(L)}{k_{0}}\left(2+\cos \theta \frac{(2 \rho+1)}{[\rho(\rho+1)]^{1 / 2}}\right) .
\end{aligned}
$$

These nonlinear coupled stochastic differential equations can be converted into an equation for the probability density $P(\rho, \theta, L)$ by use of the well-known van Kampen lemma [van Kampen 1976]. To this end we introduce a spread of "phase points" of density $Q(\rho, \theta, L)$ in $(\rho, \theta)$ "phase space" evolving in $L$ according to (5a) and (5b), subject to the initial condition that $\rho(L)=0$ at $L=0$. The phase fluid will now evolve according to the stochastic Liouville equation,

$$
\frac{\partial Q}{\partial L}=-\frac{\partial}{\partial \rho}\left(Q \frac{\partial \rho}{\partial L}\right)-\frac{\partial}{\partial \theta}\left(Q \frac{\partial \theta}{\partial L}\right),
$$

where the velocities $(\partial \rho / \partial L)$ and $(\partial \theta / \partial L)$ are given by $(5 \mathrm{a})$ and $(5 \mathrm{~b})$, respectively. We have the well-known result [van Kampen 1976] that

$$
P(\rho, \theta, L)=\langle Q(\rho, \theta, L)\rangle_{v},
$$

where $\langle\cdots\rangle_{v}$ denotes averaging with respect to the basic random variable $V(x)$. Using (5a), (5b), (6) and (7) we get,

$$
\begin{aligned}
\frac{\partial P}{\partial L}= & -\frac{\partial}{\partial \theta}\left\langle Q\left[2 k_{0}-\frac{V(L)}{k_{0}}\left(2+\cos \theta \frac{(2 \rho+1)}{[\rho(\rho+1)]^{1 / 2}}\right)\right]\right\rangle_{v} \\
& +\frac{\partial}{\partial \rho}\left\langle Q \frac{2 V(L)}{k_{0}} \sin \theta[\rho(\rho+1)]^{1 / 2}\right\rangle_{v}
\end{aligned}
$$

Now we must evaluate $\langle Q V(L)\rangle_{v}$ appearing in the right hand side of (8). This is done by using the Novikov identity (Novikov 1965) for the functional $Q$ of the Gaussian random variable $V(L)$, namely, that;

$$
\begin{aligned}
\left\langle V\left(L^{\prime}\right) Q\right\rangle_{v} & =\int_{0}^{L}\left\langle V\left(L^{\prime}\right) V\left(L^{\prime \prime}\right)\right\rangle\left\langle\frac{\delta Q}{\delta V\left(L^{\prime \prime}\right)}\right\rangle \mathrm{d} L^{\prime}, \\
& =V_{0}^{2}\left\langle\frac{\delta Q}{\delta V(L)}\right\rangle,
\end{aligned}
$$


with $0 \leqslant L^{\prime} \leqslant L$. Here $\delta Q / \delta V(L)$ is a functional derivative and can be readily obtained [Jayannavar and Kumar 1982] from (5a), (5b) and (6) as:

$$
\begin{aligned}
\left\langle\frac{\delta Q}{\delta V(L)}\right\rangle= & \frac{\partial}{\partial \theta}\left\{\frac{1}{k_{0}}\left(2+\cos \theta \frac{(2 \rho+1)}{[\rho(\rho+1)]^{1 / 2}}\right) P\right\} \\
& +\frac{\partial}{\partial \rho}\left\{\frac{2}{k_{0}} \sin \theta[\rho(\rho+1)]^{1 / 2} P\right\} .
\end{aligned}
$$

From (8)-(10) we finally get,

$$
\begin{aligned}
\frac{\partial P(\hat{\rho}, \theta, L)}{\partial L}= & -2 k_{0} \frac{\partial P}{\partial \theta}+\frac{V_{0}^{2}}{k_{0}^{2}} \frac{\partial}{\partial \theta}\left\{\left(2+\cos \theta \frac{(2 \rho+1)}{[\rho(\rho+1)]^{1 / 2}}\right)\right. \\
& \left.\times\left[\frac{\partial}{\partial \theta}\left(2+\cos \theta \frac{(2 \rho+1)}{[\rho(\rho+1)]^{1 / 2}}\right) P+\frac{\partial}{\partial \rho}\left(2 \sin \theta[\rho(\rho+1)]^{1 / 2} P\right)\right]\right\} \\
& +\frac{V_{0}^{2}}{k_{0}^{2}} \frac{\partial}{\partial \rho}\left\{2 \operatorname { s i n } \theta [ \rho ( \rho + 1 ) ] ^ { 1 / 2 } \left[\frac{\partial}{\partial \theta}\left(\left(2+\cos \theta \frac{(2 \rho+1)}{[\rho(\rho+1)]^{1 / 2}}\right) P\right)\right.\right. \\
& \left.\left.+\frac{\partial}{\partial \rho}\left(2 \sin \theta[\rho(\rho+1)]^{1 / 2} P\right)\right]\right\} .
\end{aligned}
$$

The above equation is exact for the joint probability distribution of $\rho$ and $\theta$ and is quite difficult to treat analytically. From (11) it is readily seen that $P(\rho, \theta, L)$ will depend on at least two dimensionless parameters, namely, $k_{0} L$ and $\left(V_{0}^{2} / k_{0}^{3}\right)$. Henceforth we will concentrate on the marginal distribution function $W(\rho)=$ $\int P(\rho, \theta, L) \mathrm{d} \theta$ for the resistance alone. In the present analysis we are interested in the behaviour of the distribution function in the thermodynamic limit, i.e., the large length limit. It should be noted that in this limit the reflection coefficient $r^{2}$ approaches the value unity with probability one (or $\rho \gg 1$ ) and consequently the evolution equation (5b) for $\theta$ becomes independent of $\rho$. From (5b) it follows that for $\rho \gg 1$, we get

$$
\frac{\mathrm{d} \theta}{\mathrm{d} L}=2 k_{0}-\frac{2 V(L)}{k_{0}}(1+\cos \theta)
$$

Correspondingly, one can immediately write down the associated Fokker-Planck equation [Risken 1984; Jayannavar 1990] for the probability distribution $P_{1}(\theta, L)$ which is given by,

$$
\frac{\partial P_{1}(\theta, L)}{\partial L}=-2 k_{0} \frac{\partial P_{1}}{\partial \theta}+\left(\frac{4 V_{0}^{2}}{k_{0}^{2}}\right) \frac{\partial}{\partial \theta}\left[(1+\cos \theta) \frac{\partial}{\partial \theta}(1+\cos \theta) P_{1}\right] .
$$

Generally the phase distribution evolves towards the stationary distribution once the length of the sample is larger than that of the localization length $\xi$ (defined below). This has been shown to be the case also in the tight binding Anderson model with diagonal disorder [Stone et al 1983]. Hence, in the asymptotic limit $L \gg \xi$, one can decompose $P(\rho, \theta, L)=W(\rho, L) P_{1}^{s}(\theta)$, where $P_{1}^{s}(\theta)$ is the stationary distribution for the phase which can be obtained by setting $\left(\partial P_{1}(\theta) / \partial L\right)=0$ in (13). Almost all the earlier studies have assumed that $P_{1}^{s}(\theta)$ is uniformly distributed over $[0,2 \pi]$ (i.e., $\left.P_{1}^{s}(\theta)=1 / 2 \pi\right)$. Hence the stationary distribution of phase does not depend on any 
material parameters. We call this the random phase approximation. With this approximation one can easily write down the marginal distribution of the resistance. Using (11), after some algebra, we get

$$
\frac{\partial W(\rho, l)}{\partial l}=\frac{\partial}{\partial \rho}\left\{\left(\rho^{2}+\rho\right) \frac{\partial W}{\partial \rho}\right\}
$$

where $l$ is the dimensionless length measured in units of the localization length $(l=L / \xi)$ and $\xi=\left(k_{0}^{2} / 2 V_{0}^{2}\right)$. In the large length limit, the solution of (14) is given by a well-known log-normal distribution [Kumar 1985; Shapiro 1987] and explicit expressions for the moments have also been obtained in the earlier literature [Anderson et al 1980; Abrahams and Stephen 1980; Abrikosov 1981; Melnikov 1980; Kumar 1985; Heinrichs 1986; Rammal and Doucot 1987; Shapiro 1987]. To this end we introduce an appropriate scaling variable $u=\ln (1+\rho)$ (or $u=\ln \rho$, for $\rho \gg 1$ ). From (14) one can easily derive the evolution equations for the probability distribution $W_{1}(u, l)$ of $u$ in the asymptotic regime,

$$
\frac{\partial W_{1}(u)}{\partial l}=-\frac{\partial W_{1}}{\partial u}+\frac{\partial^{2} W_{1}}{\partial u^{2}}
$$

The solution for (15) is a Gaussian and is given by,

$$
W_{1}(u, l)=\frac{1}{\sqrt{2 \pi \Delta u^{2}(l)}} \exp \left\{-\frac{(u-\overline{u(l)})^{2}}{2 \Delta u^{2}(l)}\right\},
$$

where the mean is $\overline{u(l)}=l$ and the variance is $\overline{\Delta u^{2}(l)}=l$. Note that the mean and the variance are dependent variables. Hence in the large length limit $W_{1}(u)$ with a random phase approximation approaches the universal Gaussian distribution characterized by a single parameter $\overline{(u(l)}$ or $\overline{\left.\Delta u^{2}(l)\right)}$, i.e., the large scale distribution is characterized completely by one parameter.

It has already been pointed out that the uniform phase distribution (or random phase model) is indeed a property of a random system in the weak disorder limit [Jayannavar 1990; Stone et al 1983; Heinrichs 1990] and naturally leads to the single parameter scaling theory in the weak disorder limit. This can be understood physically as follows: in the case of weak disorder the localization length can be arbitrarily large. The electron, therefore, samples a very large number of scattering sites (or penetrates deep inside the sample), thereby strongly randomizing the phase of the reflected electron. In the strong disorder limit the electron cannot sample the random potential effectively. In the strong disorder limit, the stationary phase distribution is nonuniform and its nature is sensitive to the statistics of the random potential [Jayannavar 1990; Stone et al 1983]. We will now derive the analytical expression for the stationary distribution of $\theta$ by setting $\left(\partial P_{1} / \partial L\right)=0$ in (13). We would like to point out a technical problem involved in evaluating the stationary distribution. In (13) the diffusion term, namely, the coefficient in front of the highest derivative $\left(\partial^{2} / \partial \theta^{2}\right)$, can vanish for particular values of $\theta$ and this consequently leads to divergences in the stationary distribution. This difficulty is generally encountered for Fokker-Planck equations arising from multiplicative Gaussian white noise terms [Schleich and Scully 1988] in the original Langevin type equations. For the case when noise is correlated such divergences do not appear. To avoid this difficulty one generally introduces an 
additional new diffusion coefficient $\varepsilon$ and finally at the end of the calculations one lets $\varepsilon \rightarrow 0$ (see Schleich and Scully 1988 for details). Now following closely the method of Schleich and Scully one can write down the expression for $P_{1}^{s}(\theta)$ as:

where

$$
P_{1}^{s}(\theta)=\lim _{\varepsilon \rightarrow 0} N P(\theta, \varepsilon)
$$

and

$$
\begin{gathered}
P(\theta, \varepsilon)=e^{-\phi(\theta)} \int_{\theta}^{\theta+2 \pi} \mathrm{d} \theta^{\prime} \frac{e^{-\phi\left(\theta^{\prime}\right)}}{\left[\varepsilon+\left(4 V_{0}^{2} / k_{0}^{2}\right)\left(1+\cos \theta^{\prime}\right)^{2}\right]}, \\
\phi(\theta)=\int_{-\pi}^{\theta} \mathrm{d} \theta^{\prime} \frac{\left[2 k_{0}+\left(4 V_{0}^{2} / k_{0}^{2}\right) \sin \theta^{\prime}\left(1+\cos \theta^{\prime}\right)\right]}{\left[\varepsilon+\left(4 V_{0}^{2} / k_{0}^{2}\right)\left(1+\cos \theta^{\prime}\right)^{2}\right]},
\end{gathered}
$$

$$
N=\left[\int_{\pi}^{\pi} \mathrm{d} \theta P(\theta, \varepsilon)\right]^{-1} .
$$

In particular, we note that $P_{1}^{s}(\theta)$ depends on the material parameters through the dimensionless variable $A=\left(2 V_{0}^{2} / k_{0}^{3}\right)$ where ' $A$ ' being small and large compared to unity implies weak and strong disorder respectively. In figures 1-4 we have plotted $P_{1}^{s}(\theta)$ for various values of parameters $A$ using the numerical method of continued

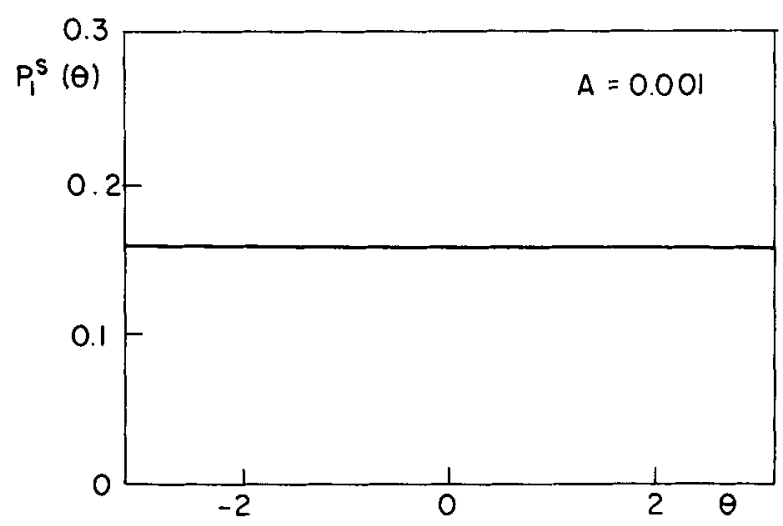

Figure 1.

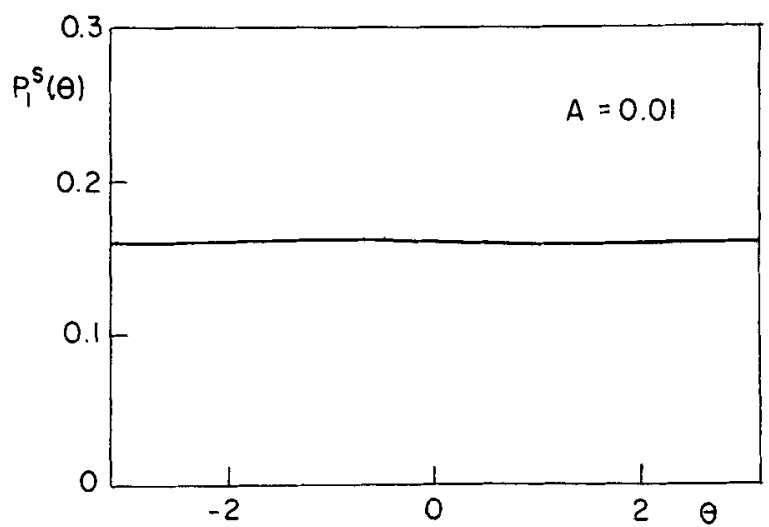

Figure 2. 


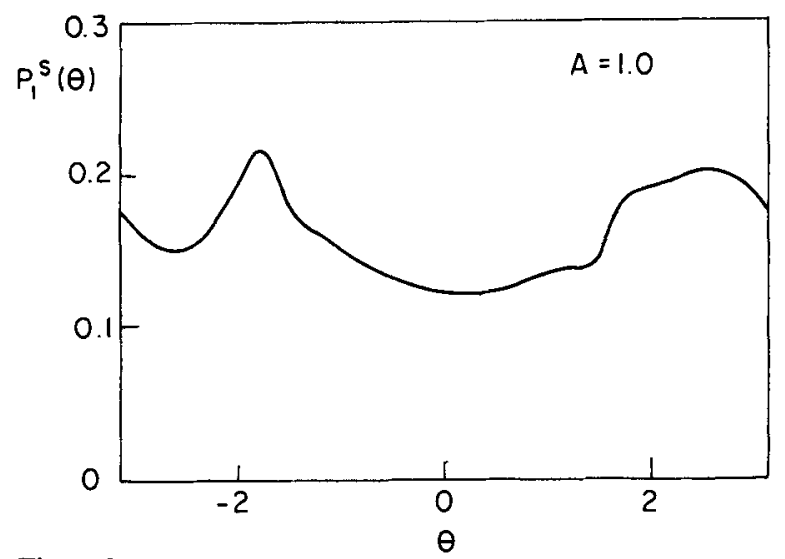

Figure 3.

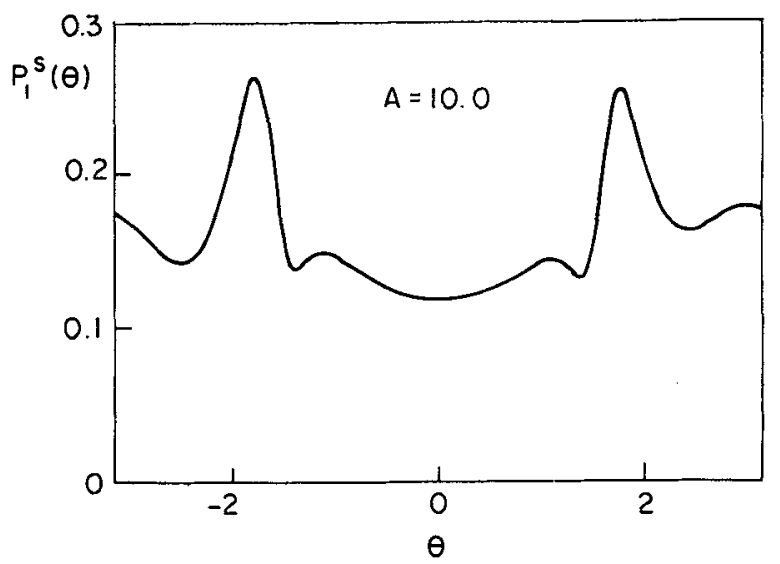

Figure 4.

Figures 1-4. Plot of $P_{1}^{s}(\theta)$ as a function of $\theta$ in the interval $-\pi$ to $+\pi$. The values of the system parameter $A$ are indicated on the graphs.

fractions [Risken 1984]. In this method we write an expansion for the stationary distribution in Fourier series, $P_{1}^{s}(\theta)=(1 / 2 \pi) \sum_{n=-\infty}^{\infty} C_{n} \exp (i n \theta)$. Substituting this in (13), with the right hand side equal to zero, we get the recurrence relation for the expansion coefficients in which only coefficients from $C_{n-2}$ to $C_{n+2}$ are coupled. Now by defining the vector $\mathrm{C}_{n}=\left(C_{2 n}, C_{2 n+1}\right)$, the recurrence relation can be written in the form of tridiagonal vector recurrence relation. Further procedure for evaluating all the vectors $C_{n}$ (or coefficients $C_{n}$ ) involves a repeated application of the matrix continued fractions (for details see Jayannavar 1990). It is clearly seen that $P_{1}^{s}(\theta)$ is indeed distributed uniformly in the weak disorder limit (Figures 1 and 2), while it is non-uniform in the strong disorder limit (Figures 3 and 4). Having established the fact that $P_{1}^{s}(\theta)$ is in general non-uniform we again carry out the same procedure as mentioned earlier for the marginal distribution function. By setting $P(\rho, \theta, l)=$ $W_{1}(\rho, l) P_{1}^{s}(\theta)$ in (11) and after some algebra, the large scale distribution for the variable $u(\sim \ln \rho)$ is given by the differential equation,

$$
\frac{\partial W(u, l)}{\partial l}=-2[(1-x)+y] \frac{\partial W}{\partial u}+2 x \frac{\partial^{2} W}{\partial u^{2}}
$$


where $x=\left\langle\sin ^{2} \theta\right\rangle_{\theta}$ and $y=\langle\cos \theta\rangle_{\theta}$. The angular brackets $\langle\cdots\rangle_{\theta}$ denote the averaging over the function of $\theta$ with respect to the stationary distribution $P^{s}(\theta)$. The solution for $W$ is given by $(16)$ with mean $\overline{u(l)}=2[(1-x)+y] l$ and variance $\overline{\Delta u^{2}}(l)=2 x l$. Again in the strong disorder limit the distribution function for the variable $u$ approaches the universal Gaussian distribution. Variables $x$ and $y$ appearing in the expressions for $\overline{u(l)}$ and $\overline{\Delta u(l)^{2}}$ depend on the material parameter $A$ and $l$. This in general leads to a two-parameter scaling theory of localization. Both $\overline{u(l)}$ and $\overline{\Delta u^{2}}(\bar{l})$ depend explicitly on $l$ and implicitly on $A$. We now check for the validity of single parameter scaling (i.e., to show $\overline{u(l)}$ and $\overline{\Delta u^{2}(l)}$ are dependent variables) for which the necessary and sufficient condition is that the Jacobian $J\left(\bar{u}, \overline{\Delta u^{2}}\right) /(l, A)$, should vanish identically [Kumar 1983], i.e.

$$
\frac{\partial\left(\bar{u}, \overline{\Delta u^{2}}\right)}{\partial(l, A)}=\left|\begin{array}{cc}
\frac{\partial \bar{u}}{\partial l} & \frac{\partial \bar{u}}{\partial A} \\
\frac{\partial \overline{\Delta u^{2}}}{\partial l} & \frac{\partial \overline{\Delta u^{2}}}{\partial A}
\end{array}\right|=0 .
$$

This gives the differential equation

$$
(1+y) \frac{\mathrm{d} x}{\mathrm{~d} A}=x \frac{\mathrm{d} y}{\mathrm{~d} A}
$$

the solution of which is given by $[x /(1+y)]=$ constant independent of $A$. We have computed $x$ and $y$ using the known coefficient $C_{n}$ in the Fourier expansion of $P_{1}^{s}(\theta)$ and we have verified numerically that indeed $[x /(1+y)]$ does depend on $A$ in the strong disorder limit. We therefore arrive at the two parameter scaling theory of localization with the full probability distribution characterized by two independent parameters.

\section{Concluding remarks}

We would like to state that our basic model at the microscopic Hamiltonian level has only two system dependent dimensionless parameters, $l$ and $A$. This is a minimal case for which we obtain a two parameter scaling in the strong disorder limit. We can in principle introduce several other independent parameters in the basic microscopic Hamiltonian through the statistical distribution of random potential, e.g. (i) a Gaussian correlated potential in that the correlation length enters as an additional parameter, (ii) a non-Gaussian potential with several independent moments. In the above cases it is not clear whether the large scale distribution function for the variable $u$ approaches the universal Gaussian distribution with two independent parameters or some other distribution function characterized by several independent parameters (many parameter scaling!). At present it is possible to obtain the closed Fokker-Planck type equations for joint distribution of $\rho$ and $\theta$ only for the dichotomic correlated (binary) random potentials [Rammal and Doucot 1987]. Generalization along these directions may still prove fruitful.

Finally we remark on the analogy with phase transition. By two parameter scaling all we mean here is that the probability distribution of $\ln \rho$ cannot be described by 
the single parameter namely $\langle\ln \rho\rangle$ even in the limit of infinite length (the so called "thermodynamic limit"). One needs to specify independently the variance also. In contrast the one parameter scaling would require that the distribution be characterized entirely in terms of $\langle\ln \rho\rangle$. It should be noted that otherwise tempting analogy with phase transition (scaling behaviour) is somewhat misleading here in that there is no phase transition in the one-dimensional case, i.e., there is no localized to delocalized transition. The question of scaling behaviour in the sense of phase transition can be addressed only in higher dimension where there is a mobility edge (fixed point). However, we do not have the analogous probabilistic treatment for higher dimensions at present.

\section{Acknowledgment}

The author would like to thank Professor N Kumar for many useful discussions.

\section{References}

Abrahams E, Anderson P W, Licciardello D C and Ramakrishnan T V 1979 Phys. Rev. Lett. 42673

Abrahams E and Stephen M 1980 J. Phys. C13 L377

Abrikosov A A 1981 Solid State Commun. 37997

Altshuler B L and Khmelnitskii D E 1985 Pis'ma Zh. Eksp. Teor. Fiz. 42291 [JETP Lett. 198542 359]

Altshuler B L, Kravtsov V E and Lerner I V 1986 Pis'ma Zh. Eksp. Teor. Fiz. 43342 [JETP Lett. 1986 $43441]$

Anderson P W, Thouless D J, Abrahams E and Fisher D S 1980 Phys. Rev. B22 3519

Bellman R and Wing G M 1976 An introduction to invariant imbedding (New York: Wiley)

Bishop D J and Dolan G J 1985 Phys. Rev. Lett. 552911

Chandrasekhar S 1960 Radiative transfer (New York: Dover)

Cohen A, Roth Y and Shapiro B 1988 Phys. Rev. B38 12125

Efetov K B 1984 Pis'ma Zh. Eksp. Teor. Fiz. 4017 [JETP Lett. 198440738 ]

Erdos P and Herndon R C 1982 Adv. Phys. 3163

Farrell M E, Bishop M F, Kumar N and Lawrence W E 1985 Phys. Rev. Lett. 55626

Giordano N 1980 Phys. Rev. B22 5635

Heinrichs J 1986 Phys. Rev. B33 5261

Heinrichs J, Jayannavar A M and Kumar N 1988 Solid State Commun. 671103

Heinrichs J 1990 J. Phys. Condens. Matter, 21559

Ioffe L B, Sagdeev I R and Vinokur V M 1985 J. Phys. C18 L641

Jayannavar A M and Kumar N 1982 Phys. Rev. Lett. 48553

Jayannavar A M 1990 Solid State Commun. 73247

Kumar N 1983 J. Phys. C16 L109

Kumar N 1985 Phys. Rev. B31 5513

Kumar N and Jayannavar A M 1986 J. Phys. C19 L85

Landauer R 1970 Philos. Mag. 21863

Lee P A and Stone A D 1985 Phys. Rev. Lett. 551622

Melnikov V I 1980 Zh. Eksp. Teor. Fiz. Pis'ma 32244 [JETP Lett. 198032 225]

Novikov E A 1964 Zh. Eksp. Teor. Fiz. 471919 [Sov. Phys. JETP 196520 1290]

Rammal R and Doucot B 1987 J. Phys. (Paris) 48509

Risken H 1984 The Fokker-Planck equations (Berlin: Springer)

Schleich W and Scully M O 1988 Phys. Rev. A37 1261

Shapiro B 1987 Philos. Mag. 561031

Stone A D, Allan D C and Joannopoulos J D 1983 Phys. Rev. B27 836

van Kampen N G 1976 Phys. Rep. C24 172 